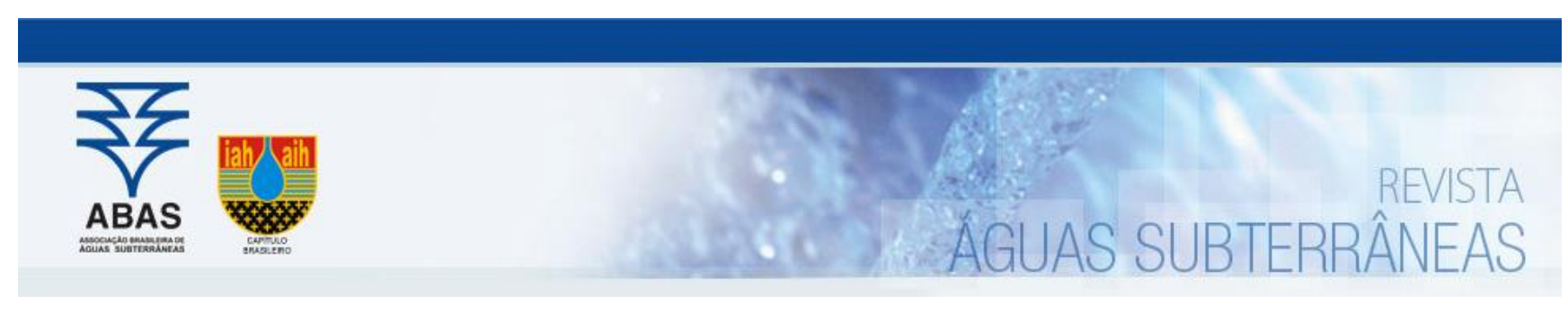

Artigos

\title{
0 uso da ferramenta CALPOHID e de valores diferentes de recarga na avaliação do comprometimento e do potencial hídrico subterrâneo de bacias hidrográficas
}

\section{The use of the CALPOHID tool and different recharge values in the assessment of water basin impairment and groundwater potential of hydrographic basins}

\author{
Tuane de Oliveira Dutra1; Pedro Antonio Roehe Reginato1; Taison Anderson Bortolin² \\ 1 Instituto de Pesquisas Hidráulicas (UFRGS), Porto Alegre, RS \\ 2 Instituto de Saneamento Ambiental (UCS), Caxias do Sul, RS \\ $\triangle \underline{\text { tuanehidrica@gmail.com, pedro.reginato@ufrgs.br, taisonbortolin@yahoo.com.br }}$
}

\section{Palavras-chave:}

Bacia hidrográfica Taquari-Antas. Gestão dos recursos hídricos. Disponibilidade hídrica instalada.

Keywords:

Taquari-Antas hydrographic basin. Water resources management. Installed water availability

Revisão por pares.

Recebido em: 06/06/2020. Aprovado em: 29/01/2021.

\begin{abstract}
Resumo
A estimativa do potencial hídrico subterrâneo e do comprometimento do mesmo se faz necessária para auxiliar na seleção de medidas em prol do uso sustentável do aquífero. Tais informações se tornam ainda mais relevantes em áreas com maior adensamento de poços e inseridas em sistemas aquíferos fraturados. Esse é o caso da área de estudo, que está inserida na bacia hidrográfica Taquari-Antas, onde há ocorrência do Sistema Aquífero Serra Geral (SASG). O objetivo deste estudo foi o de analisar a variação do volume do potencial hídrico subterrâneo (Po) e do comprometimento do mesmo (C), à frente de 5 métodos de estimativa da recarga: 2 variações de métodos de balanço hídrico e 3 métodos baseados em águas superficiais. Para tanto, foi desenvolvida uma ferramenta de cálculo do Po e C, denominada CALPOHID, onde foram simulados 5 cenários de potencial, considerando-se 15, 20, 25, 50 e 100\% da recarga, e 3 cenários de disponibilidade hídrica instalada (Di), considerando os tempos de bombeamento médio de 18 e $24 \mathrm{~h}$. Na maioria dos casos ocorreram diferenças significativas entre os métodos com capacidade de atenderem, no mínimo, $42 \%$ o valor de Di em seu cenário mais crítico. Contudo, tais diferenças só resultaram em variações no grau de comprometimento para os cenários de potencial de 15, 20 e $25 \%$ da recarga. Logo, o método do balanço hídrico (BH1), foi o que resultou nas menores diferenças em relação aos demais métodos, propiciando um caminho ponderado entre os 5 métodos simulados. A ferramenta CALPOHID demonstrou ser eficiente para a comparação de cenários de potencial e comprometimento diante de diferentes dados de recarga e Di.
\end{abstract}

Abstract

The estimation of groundwater potential and its commitment are necessary to assist in the selection of measures for the sustainable use of the aquifer. Such information becomes even more relevant in areas with greater density of wells and inserted in fractured aquifer systems. This is the case of the study area, which is inserted in the TaquariAntas hydrographic basin, where the Serra Geral Aquifer System (SASG) occurs. The aim of this study was to analyze the variation in the volume of groundwater potential (Po) and its impairment (C), in view of 5 methods of recharge estimation: 2 variations of water balance methods and 3 methods based on surface water. For this, a Po and C calculation tool, called CALPOHID, was developed in which 5 potential scenarios were simulated, considering 15, 20, 25,50 and $100 \%$ of the recharge, and 3 scenarios of installed hydric availability (Di), considering the average pumping times of 18 and $24 \mathrm{~h}$. In most cases, there were significant differences between the methods capable of meeting at least $42 \%$ of the Di value in its most critical scenario. However, such differences only resulted in variations in the degree of impairment for the potential scenarios of 15,20 and $25 \%$ of the recharge. Therefore, the water balance method (BH1) was the one that resulted in the smallest differences in relation to the other methods, providing a weighted result between the 5 simulated methods. The CALPOHID tool proved to be efficient for comparing potential and commitment scenarios in the view of different recharge and Di data.

\section{INTRODUÇÃO}

$\mathrm{Na}$ ausência de dados quantitativos do aquífero a gestão desse é desenvolvida de forma precária, prejudicando a apli- cação de medidas que propiciem o uso sustentável dos recursos hídricos subterrâneos. Logo, a avaliação do potencial hídrico subterrâneo e do grau de comprometimento do mesmo é de suma importância para o planejamento das medidas de 
gestão.

Conforme Todd (1959), o potencial do aquífero é definido como sendo a vazão anual que pode ser explotada do aquífero sem que se produza um efeito indesejável. Além disso, o potencial depende diretamente da reserva renovável e permanente existente. De acordo com Costa (1998), o potencial é o volume de água acumulado no meio aquífero em função da porosidade eficaz ou do coeficiente de armazenamento, sendo variável anualmente em decorrência dos aportes sazonais de água superficial, do escoamento subterrâneo e dos exutórios. Desta forma, o potencial pode consistir em um \% ou na totalidade da recarga. Ainda não há um valor fixo do percentual que deve ser utilizado para se determinar o volume de água disponível em bacias hidrográficas, sendo que, a depender dos autores, diferentes valores são usados. Conforme ANA (2005), tal dificuldade é devido à dinâmica de fluxo e a resposta de cada aquífero à explotação. Ainda segundo a agência, a dificuldade pode ser maior ou menor em relação ao nível de conhecimento sobre o sistema aquífero e dos fatores ambientais, climáticos e locais envolvendo o mesmo. ANA (2005) utilizou o valor de $20 \%$ do volume das reservas reguladoras, sendo considerada pela mesma uma porcentagem conservadora, que não utiliza as reservas permanentes. Rebouças et al. (1994), na realização do diagnóstico hidrogeológico da Região Metropolitana de São Paulo, utilizaram 25\% das reservas reguladoras, devido a descontinuidade da zona aquífera e a grande heterogeneidade dos sedimentos. DAEE (2009), em estudo para a elaboração do plano diretor de aproveitamento de recursos hídricos para a macro metrópole paulista, utilizou o percentual de $50 \%$ das reservas reguladoras. Já Costa (1998), recomendou para aquíferos fissurais a utilização de $15 \%$ das reservas reguladoras.

A avaliação do potencial e do percentual de comprometimento deste, a qual consiste em avaliar quanto do potencial hídrico subterrâneo já está sendo utilizado através da disponibilidade hídrica instalada, ganha maior relevância em regiões com maior dependência desses recursos e ocorrência de aquíferos fraturados, como o Sistema Aquífero Serra Geral (SASG). Conforme Reginato e Strieder (2004), as características hidrodinâmicas do SASG evidenciam um comportamento fortemente anisotrópico, e em função disso a potencialidade do aquífero é intensamente variada. Logo, a bacia hidrográfica TaquariAntas está enquadrada em ambas as situações, com mais de 90\% de sua área com ocorrência de aquíferos fraturados (SASG) e com quase $70 \%$ dos municípios sendo abastecidos exclusivamente por água subterrânea.

Com o objetivo de se avaliar a variação do potencial e do percentual de comprometimento do SASG na bacia hidrográfica Taquari-Antas desenvolveu-se este estudo. A avaliação foi realizada para uma sub-bacia, com base no uso de diferentes dados de recarga e disponibilidade hídrica instalada. Sendo essa última associada a água passível de ser consumida através das captações de água já existentes (Costa, 1998). Para tanto, foi desenvolvida neste estudo a ferramenta de cálculo do potencial hídrico subterrâneo (CALPOHID), visando auxiliar a gestão dos recursos hídricos subterrâneos.

\section{2. ÁREA DE ESTUDO}

Para o desenvolvimento deste estudo foi selecionada uma sub-bacia inserida na bacia hidrográfica Taquari-Antas, onde há ocorrência do Sistema Aquífero Serra Geral e o abastecimento de água, na maior parte da área, é realizado por meio da captação de água subterrânea. Além disso, a área em questão tem estudos de recarga realizados por mais de um tipo de método.

A área de estudo está localizada no Alto Rio Carreiro, na parte mais superior da Unidade de gestão do rio Carreiro (UC). A área em questão, possuí $1330 \mathrm{~km}^{2}$, correspondendo a $5 \%$ da área total da bacia Taquari-Antas, conforme apresentado na figura 1.

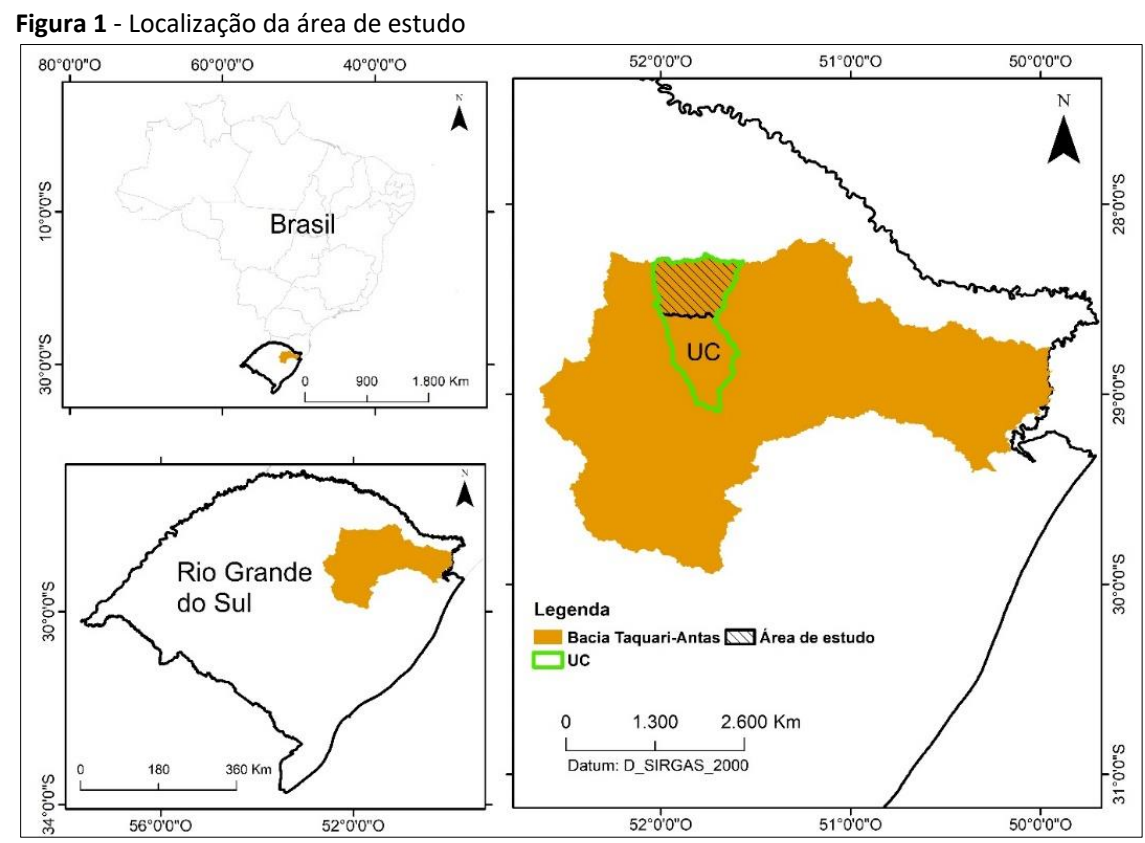


A área de estudo está toda inserida no Sistema Aquífero Serra Geral II, o qual, segundo Machado \& Freitas (2005), possuí capacidades específicas inferiores a $0,5 \mathrm{~m}^{3} / \mathrm{h} / \mathrm{m}$, podendo ser encontrado em áreas mais fraturadas ou com arenitos na base do sistema com valores superiores a $2 \mathrm{~m}^{3} / \mathrm{h} / \mathrm{m}$, sendo classificado como um aquífero com média a baixa possibilidade de ocorrência de água subterrânea. Na área em questão há duas classes de solos: Latossolos Vermelhos (Nitossolos) e Neossolos litólicos associados com Chernossolos Argilúvicos (STRECK et al. 2008), sendo que, o principal uso do solo na região é o agrícola (57,6\% da área), seguido por mata nativa, com cerca de $25 \%$.

\section{METODOLOGIA}

\subsection{Recarga}

A recarga utilizada para a estimativa do potencial foi calculada por Bortolin (2018) na área de estudo, sendo que para esse estudo foram utilizadas 2 variações do método de balanço hídrico e 3 métodos baseados na separação de escoamento de base, conforme apresentado abaixo:

- $\quad$ BH1: método baseado nos estudos de Wanfried e Hirata (2005), que fizeram uma adaptação do modelo de Fenn et al. (1975).

- BH4: método da curva número (CN) do US Soil Conservation Service (NRCS, 2004).

- $\quad$ Filtro com um único parâmetro (FP1), proposta inicialmente por Lyne e Hollick (1979);

- Filtro com dois parâmetros (FP2), proposta por Eckhardt (2005);

- Filtro inverso $(\mathrm{FI})$, proposta por Collischonn e Fan (2013).

Os métodos citados acima foram selecionados por serem os mais amplamente utilizados. A formulação dos métodos em questão é apresentada no quadro 1 abaixo:

\begin{tabular}{|c|c|c|}
\hline Autores & \multicolumn{2}{|l|}{ Métodos } \\
\hline \multirow{3}{*}{$\begin{array}{l}\text { Wanfried e Hirata } \\
\text { (2005), adaptado de } \\
\text { Fenn et al. (1975) }\end{array}$} & \multicolumn{2}{|l|}{$\mathrm{BH} 1$} \\
\hline & Roff = C'.P & (1) \\
\hline & $C^{\prime}=\alpha \cdot C$ & (2) \\
\hline \multirow{3}{*}{ NRCS (2004) } & \multicolumn{2}{|l|}{$\mathrm{BH} 4$} \\
\hline & Roff $=\frac{(P-0,2 S)^{2}}{P+0,8 S}$ Se $P>0,2 S$ & (3) \\
\hline & $S=\frac{25400}{C N}-254$ & (4) \\
\hline \multirow[b]{2}{*}{ Lyne e Hollick (1979) } & \multicolumn{2}{|l|}{ FP1 } \\
\hline & $b_{i}=\alpha b_{i-1}+\frac{(1-\alpha)}{2} \cdot\left(y_{i}+y_{i-1}\right)$ & (5) \\
\hline \multirow[b]{2}{*}{ Eckhardt (2005) } & \multicolumn{2}{|l|}{ FP2 } \\
\hline & $\mathrm{b}_{\mathrm{i}}=\frac{\left(1-\mathrm{BFI}_{\max }\right) \alpha \cdot \mathrm{b}_{\mathrm{i}-1}+(1-\alpha) \cdot \mathrm{BFI}_{\max } \cdot y_{i}}{1-\alpha \cdot \mathrm{BFI}_{\max }}$ & (6) \\
\hline \multirow{3}{*}{$\begin{array}{l}\text { Collischonn e Fan } \\
\text { (2013) }\end{array}$} & \multicolumn{2}{|l|}{$\mathrm{FI}$} \\
\hline & $b_{i-1}=\frac{b i}{\alpha}$ & (7) \\
\hline & $B F I_{\max }=\frac{\sum_{i=1}^{N} b^{\prime} i}{\sum_{i=1}^{N} y_{i}}$ & (8) \\
\hline
\end{tabular}


Onde:

C: coeficiente que determina o quanto de água precipitada escorrerá em superfície, em função do litotipo. Conforme Bortolin (2018), C = 0,45;

$\alpha$ : é determinado em função da declividade e da estação do ano, seca ou úmida. Conforme Bortolin (2018), $\alpha=0,70$ (estação seca) e 0,85 (estação úmida);

Roff: escoamento superficial;

P: precipitação $(\mathrm{mm})$;

S: potencial máximo de retenção $(\mathrm{mm})$;

0,2 S: perdas iniciais por interceptação, infiltração e reten-

ção superficial;

$\mathrm{CN}$ : Número da Curva;

bi: escoamento de base no tempo i;

$b$ i-1: escoamento de base no tempo i-1;

$y i$ : escoamento total no tempo i;

$y i-1$ : escoamento total no tempo i-1;

$\alpha$ : parâmetro do filtro (estimado em 0,925 por Nathan e

McMahon ,1990 e Arnold et al.,1995);

BFImax: máximo valor do índice de escoamento de base.

Para maiores detalhamentos sobre os métodos e suas limitações orienta-se que se realize a leitura do trabalho de Bortolin (2018). No quadro 2 são apresentados os \% de recarga que foram utilizados nesse estudo, os quais foram obtidos por Bortolin (2018) com a aplicação das metodologias descritas acima.

Quadro 2 - \% de recarga em relação a precipitação estimado por Bortolin (2018)

\begin{tabular}{|c|c|}
\hline Método & \% da precipitação \\
\hline BH1 & 24,9 \\
\hline BH4 & 36,8 \\
\hline FP1 & 19,2 \\
\hline FP2 & 10,48 \\
\hline Fl & 19,96 \\
\hline
\end{tabular}

\subsection{Ferramenta de auxílio à gestão dos recursos hídricos sub- terrâneos - CALPOHID}

CALPOHID é uma ferramenta de cálculo que foi desenvolvida em planilha eletrônica, no programa Excel, e tem como finalidade auxiliar o gestor na tomada de decisão diante de questões envolvendo os recursos hídricos subterrâneos. Sendo assim, o intuito da mesma é propiciar, de forma rápida e fácil, o cálculo do potencial hídrico subterrâneo, do percentual e grau de comprometimento dos recursos hídricos subterrâneos e da disponibilidade hídrica instalada (Di). 0 cálculo da Di foi realizado através das informações inseridas no banco de dados dos poços, o qual foi elaborado a partir da coleta de informações realizada junto ao Sistema de Informações de Águas Subterrâneas (SIAGAS), Sistemas de Outorga de Águas Subterrâneas do Rio Grande do Sul (SIOUT), Companhia Riograndense de Saneamento (CORSAN), Programa de Perfuração de Poços Artesianos (PAP/RS), prefeituras e empresas de perfuração.
No quadro 3, são apresentadas as fórmulas utilizadas para as estimativas do Potencial hídrico subterrâneo (Po), Disponibilidade hídrica instalada (Di), Comprometimento (C) e Volume do potencial restante $(\mathrm{V})$.

Quadro 3 - Fórmulas utilizadas na CALPOHID para a estimativa dos parâmetros

\begin{tabular}{|c|lc|}
\hline Parâmetro & \multicolumn{1}{|c|}{ Fórmula } \\
\hline $\begin{array}{c}\text { Potencial Hídrico } \\
\text { Subterrâneo (Po) }\end{array}$ & $\mathrm{Po}=(\mathrm{X} \%$.R). $\mathrm{A}$ \\
\hline $\begin{array}{c}\text { Disponibilidade hídrica } \\
\text { instalada (Di) }\end{array}$ & $\mathrm{Di}=\sum(\mathrm{Q} . \mathrm{Tb} .365)$ \\
\hline $\begin{array}{c}\text { Comprometimento de (Po) } \\
\text { Volume restante }(\mathrm{V})\end{array}$ & $\mathrm{C}=\frac{\mathrm{Di}}{\mathrm{Po}}$ \\
\hline
\end{tabular}

Onde:

X: é a porcentagem aplicada sobre a recarga (\%);

A: área da Sub-bacia $\left(\mathrm{m}^{2}\right)$;

Tb: tempo de bombeamento $(h)$;

Q: vazão $\left(m^{3} / \mathrm{h}\right)$.

Portanto, com a CALPOHID é possivel:

- Simular até 5 cenários de potencial. Neste estudo foram simulados os cenários de $15,20,25,50$ e 100 $\%$ da recarga;

- Simular até 3 cenários de Di. Neste estudo foram simulados os cenários com Tb médio de 18 e 24h;

- Inserir até 5 dados de recarga e avaliar a variação dos quadros de comprometimento diante dos mesmos;

- Verificar a alteração nos quadros de comprometimento diante das novas solicitações de captação. Neste trabalho não se realizou esta análise;

- Verificar o volume restante do potencial hídrico subterrâneo diante de cada cenário;

- Realizar as análises mencionadas para um período anual e mensal. Neste estudo realizou-se apenas a análise anual.

Levando em consideração a parcela de poços não cadastrados na bacia, o plano de bacia aplicou um percentual de $50 \%$ de aumento sobre o valor da Di calculada, para que a mesma se aproximasse de volumes mais realistas, levando-se em consideração a existência de poços clandestinos que não estão cadastrados em nenhum sistema (STE, 2011). Sendo assim, neste estudo também foi admitido o valor de $50 \%$ sobre o valor total calculado de Di para os 3 cenários.

A estimativa do comprometimento do potencial hídrico subterrâneo (C) foi realizada através da equação 9, apresentada anteriormente, e considerando a classificação dos graus de comprometimento determinados na Deliberação CRH n52/2005, apresentado no quadro 4. 
Quadro 4 - Classificação do comprometimento do Potencial hídrico subterrâneo

\begin{tabular}{|c|c|c|}
\hline \multicolumn{3}{|c|}{ Nível de comprometimento } \\
\hline $50 \%>$ (Dit/Po) & $50 \% \leq$ (Dit/Po) $<75 \%$ & $\begin{array}{c}(\text { Dit/Po }) \geq \\
75 \%\end{array}$ \\
\hline Baixo & Médio & Crítico \\
\hline
\end{tabular}

Cabe salientar que a CALPOHID pode ser utilizada em outras áreas (bacias, sub-bacias, municípios, etc) além da utilizada nesse trabalho. Também deve-se destacar que a ferramenta não realiza a estimativa da recarga, o dado em questão deve ser estimado utilizando a metodologia e a escala que melhor esteja adequada a área de estudo ou aos dados disponíveis na mesma.

Além da recarga, para utilizar a CALPOHID são necessários os seguintes dados:
- Tamanho da área de estudo $\left(\mathrm{Km}^{2}\right)$;

- Precipitação média anual e/ou mensal (mm/ano ou mês);

- $\quad \%$ de recarga em relação a precipitação;

- Quantidade de poços existentes na área de estudo;

- Tempo de bombeamento médio (h);

- Vazão média ou mediana $\left(m^{3} / h\right)$.

Para facilitar a inserção dos dados e visualização dos resultados, a CALPOHID foi dividida em 5 módulos, que são apresentados no quadro 5 . Sendo que somente no módulo 1 é feita a inserção dos dados, nos demais é realizada a análise dos resultados perante aos diferentes cenários simulados.

No módulo 4 é aplicado um condicional para que somente os valores de volume restante que foram inferiores a zero fiquem com a cor vermelha nos gráficos gerados no módulo 5 .

Quadro 5 - Descrição dos módulos da CALPOHID

\begin{tabular}{|l|l|}
\hline \multicolumn{1}{|c|}{ Módulo } & \\
\hline Módulo 1 & $\begin{array}{l}\text { - Identificação da área simulada; } \\
\text { - Inserção dos dados necessários; } \\
\text { - Estimativas dos volumes de Potenciãa e Di. }\end{array}$ \\
\hline Módulo 2 & Resultado dos \% e graus de comprometimento referentes aos diversos cenários \\
\hline Módulo 3 & Volumes restantes dos potenciais hídricos subterrâneos \\
\hline Módulo 4 & Aplicação de um condicional para confecção dos gráficos de volume restante. \\
\hline Módulo 5 & Apresentação dos 5 gráficos de volume restante \\
\hline
\end{tabular}

\section{RESULTADOS E DISCUSSÕES}

\subsection{Caracterização hidrogeológica}

Através do banco de dados elaborado foram identificados 344 poços na área de estudo, os quais se encontram relativamente bem distribuídos, conforme apresentado na figura 2 . Sendo possível observar uma maior quantidade de poços nos municípios de David Canabarro e Ciríaco.

Figura 2 - Distribuição de poços na área de estudo

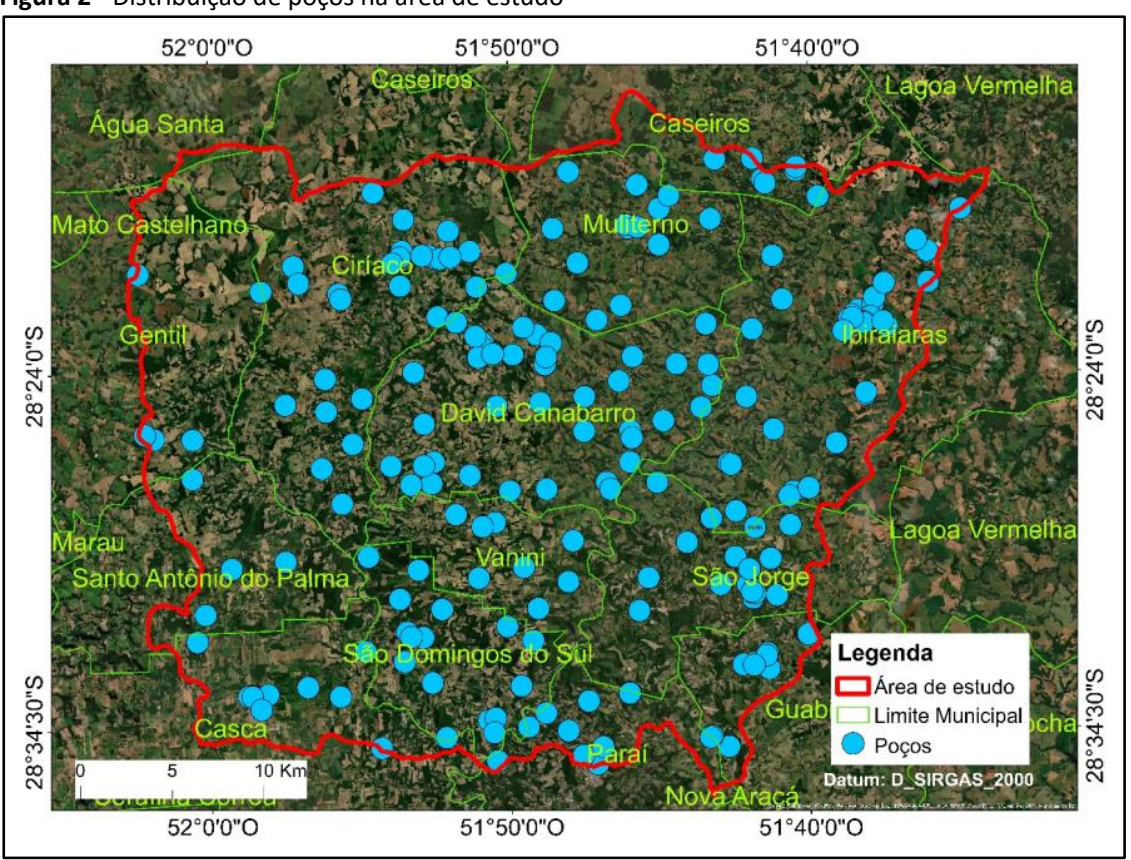


Os dados hidrodinâmicos dos poços da região estão apresentados na tabela 1, na qual é possível observar que os dados de vazão são relativamente baixos, com média e mediana inferiores a 12 e $8 \mathrm{~m}^{3} / \mathrm{h}$, respectivamente. Contudo, devido as características anisotrópicas dos aquíferos fraturados, os dados de vazão apresentaram um valor de desvio padrão significativo, o qual é justificado pelos valores mínimos e máximos de vazão apresentados. 0 mesmo foi observado nos dados de nível estático (NE) dos poços, que também apresentaram um valor de desvio padrão elevado, com o valor mínimo sendo inferior a 0,3 m e o máximo superior a 100m. 0 tempo de bombeamento apresentou pouca variação, sendo em média de 12a $24 \mathrm{~h}$, sendo o valor mediano ligeiramente inferior a $12 \mathrm{~h}$.

Tabela 1 - Dados hidrodinâmicos dos poços

\begin{tabular}{cccccc}
\hline \multirow{2}{*}{ Parâmetro } & Média & Mediana & $\begin{array}{c}\text { Análise estatística } \\
\text { Desvio padrão }\end{array}$ & Mínimo & Máximo \\
\hline Vazão $\left(\mathrm{m}^{3} / \mathrm{h}\right)$ & 11,90 & 7,80 & 12,84 & 1,20 & 66,00 \\
$\mathrm{NE}(\mathrm{m})$ & 15,85 & 9,01 & 17,70 & 0,22 & 102,37 \\
$\mathrm{~Tb}(\mathrm{~h})$ & 12,24 & 12,00 & 2,63 & 8,00 & 18,00 \\
\hline
\end{tabular}

Cabe salientar que os dados apresentados na tabela 1 para a região de estudo são coerentes com outros estudos (Reginato \& Strieder, 2006; Freitas et al 2012; Dias, 2013; Bortolin, et al 2016 e Dutra, 2019) desenvolvidos na região nordeste do estado do RS, no sistema aquífero Serra Geral.

\subsection{Disponibilidade Hídrica Instalada (Di) e Potencial hídrico Subterrâneo (Po)}

As estimativas de Di e Po foram realizadas no módulo 1 da CALPOHID. Devido à grande extensão do módulo 1, o mesmo será apresentado nos quadros 6 e 7 .
Os valores de potenciais referente a cada método de estimativa da recarga foram, em geral, distintos. Sendo, para o cenário de $100 \%$, de no máximo $942,17 \mathrm{hm}^{3}$, referente a recarga estimada com BH4 e no mínimo de 40,25 hm $^{3}$ com o método FP2 (Quadro 6). Os métodos que resultaram nos volumes de potencial mais próximos foram FP1 e FI, sendo esse ligeiramente superior, com a recarga estimada por $\mathrm{Fl}$ de 511,03 $\mathrm{hm}^{3}$, ante $491,57 \mathrm{hm}^{3}$ com o dado de FP1 (Quadro 6). Devido ao maior \% de recarga estimado pelos métodos do balanço hídrico, os volumes de potencial considerando tais métodos foram superiores aos gerados com os métodos com base em filtros digitais.

Quadro 6 - Dados de entrada e estimativa do potencial no módulo 1

\begin{tabular}{|c|c|c|c|c|c|}
\hline \multicolumn{6}{|c|}{ Módulo 1} \\
\hline Área de estudo & \multicolumn{5}{|c|}{ Sub-bacia do Alto Rio Carreiro } \\
\hline R1 & \multicolumn{5}{|c|}{$\mathrm{BH} 1$} \\
\hline $\mathrm{R} 2$ & \multicolumn{5}{|c|}{$\mathrm{BH} 4$} \\
\hline R3 & \multicolumn{5}{|c|}{ FP1 } \\
\hline R5 & \multicolumn{5}{|c|}{$\mathrm{FI}$} \\
\hline $\mathrm{A}\left(\mathrm{Km}^{2}\right)$ & \multicolumn{5}{|c|}{1330} \\
\hline $\mathrm{P}$ (mm/ano) & \multicolumn{5}{|c|}{1925} \\
\hline \multicolumn{6}{|c|}{ Estimativa da Recarga } \\
\hline & R1 & R2 & R3 & R4 & R5 \\
\hline Cenários & Po 1 & Po 2 & Po 3 & Po 4 & Po 5 \\
\hline $15 \%$ & 95,63 & 141,33 & 73,74 & 40,25 & 76,65 \\
\hline $20 \%$ & 127,50 & 188,43 & 98,31 & 53,66 & 102,21 \\
\hline $25 \%$ & 159,38 & 235,54 & 122,89 & 67,08 & 127,76 \\
\hline $50 \%$ & 318,75 & 471,09 & 245,78 & 134,16 & 255,51 \\
\hline $100 \%$ & 637,50 & 942,17 & 491,57 & 268,31 & 511,03 \\
\hline
\end{tabular}

A diferença existente entre os volumes de potenciais estimados por cada método foi realizada considerando os dois cenários extremos de potenciais de 15 e 100\% da recarga (Tabela 2). Os métodos FP1 e FI foram os que originaram as menores diferenças entre os potenciais estimados, devido a aproximação dos valores de recarga entre os mesmos (Tabela 2).Os valores de diferenças mais elevadas ocorreram entre os poten- ciais estimados com os métodos $\mathrm{BH} 4$ e $\mathrm{FP} 2$, os quais foram superiores a 100 e 670 hm $^{3}$, considerando os cenários de 15 e $100 \%$, respectivamente. As diferenças dos potenciais estimados com os demais métodos, em relação ao estimado com $\mathrm{BH} 1$, em geral foram menores, sendo, em termos absolutos, de no máximo $45,70 \mathrm{hm}^{3}$ em relação a $\mathrm{BH} 4$ e de no mínimo $18,97 \mathrm{hm}^{3}$, em relação a $\mathrm{FI}$ (Tabela 2). 
Tabela 2 - Diferença entre os volumes de potenciais estimados pelos diferentes métodos para os cenários de 100 e $15 \%$ da recarga

\begin{tabular}{|c|c|c|c|c|c|c|c|}
\hline \multirow[b]{2}{*}{ Método } & \multirow[b]{2}{*}{$\%$ de P } & \multirow{2}{*}{ Po $\left(\mathrm{hm}^{3}\right)$} & \multicolumn{5}{|c|}{$\neq$ de Po entre os métodos } \\
\hline & & & $\mathrm{BH} 1$ & $\mathrm{BH} 4$ & FP1 & FP2 & $\mathrm{FI}$ \\
\hline & & \multicolumn{6}{|c|}{$15 \%$} \\
\hline $\mathrm{BH} 1$ & 24,9 & 95,63 & $\ldots .$. & 45,70 & $-21,89$ & $-55,38$ & $-18,97$ \\
\hline $\mathrm{BH} 4$ & 36,8 & 141,33 & $-45,70$ & $\ldots .$. & $-67,59$ & $-101,08$ & $-64,67$ \\
\hline FP1 & 19,2 & 73,74 & 21,89 & 67,59 & $\ldots .$. & $-33,49$ & 2,92 \\
\hline FP2 & 10,48 & 40,25 & 55,38 & 101,08 & 33,49 & ..... & 36,41 \\
\hline \multirow[t]{2}{*}{$\mathrm{FI}$} & 19,96 & 76,65 & 18,97 & 64,67 & $-2,92$ & $-36,41$ & $\ldots .$. \\
\hline & & & \multicolumn{5}{|c|}{$\neq$ de Po entre os métodos } \\
\hline \multirow[t]{2}{*}{ Método } & $\%$ de P & $\mathrm{Po}\left(\mathrm{nm}^{\circ}\right)$ & $\mathrm{BH} 1$ & $\mathrm{BH} 4$ & FP1 & FP2 & $\mathrm{FI}$ \\
\hline & & \multicolumn{6}{|c|}{$100 \%$} \\
\hline $\mathrm{BH} 1$ & 24,9 & 637,50 & $\cdots .$. & 304,67 & $-145,93$ & $-369,19$ & $-126,48$ \\
\hline $\mathrm{BH} 4$ & 36,8 & 942,17 & $-304,67$ & $\ldots .$. & $-450,60$ & $-673,86$ & $-431,15$ \\
\hline FP1 & 19,2 & 491,57 & 145,93 & 450,60 & $\ldots .$. & $-223,25$ & 19,46 \\
\hline FP2 & 10,48 & 268,31 & 369,19 & 673,86 & 223,25 & $\ldots .$. & 242,71 \\
\hline $\mathrm{FI}$ & 19,96 & 511,03 & 126,48 & 431,15 & $-19,46$ & $-242,71$ & $\ldots .$. \\
\hline
\end{tabular}

Com exceção da diferença entre os métodos FP1 e FI para o cenário de $15 \%$, as demais diferenças originadas foram significativas. Isso fica claro ao compará-las com os volumes de Di em seu cenário mais crítico $\left(44,97 \mathrm{hm}^{3}\right)$. Por exemplo, a diferença de $18 \mathrm{hm}^{3}$ (entre $\mathrm{BH} 1$ e Fl), no cenário de 15\%, atenderia $42 \%$ da $\mathrm{Di}$ (Tb $24 \mathrm{~h}$ ) em questão.
0 volume de disponibilidade hídrica instalada, referente aos 344 poços situados na região de estudo, não ultrapassou 30 $\mathrm{hm}^{3} /$ ano quando considerado um Tb de $24 \mathrm{~h}$, sendo de 15,46 $\mathrm{hm}^{3}$ para o cenário menos crítico de $\mathrm{Di}$, conforme apresentado no módulo 1 (Quadro 7). Quando acrescido em $50 \%$, os volumes de Di ficaram entre 25 e $45 \mathrm{hm}^{3} /$ ano.

Quadro 7 - Estimativa dos volumes de Di no módulo 1

Estimativa da Disponibilidade Hídrica Subterrâneo ( $\left.\mathrm{hm}^{3} / \mathrm{ano}\right)$

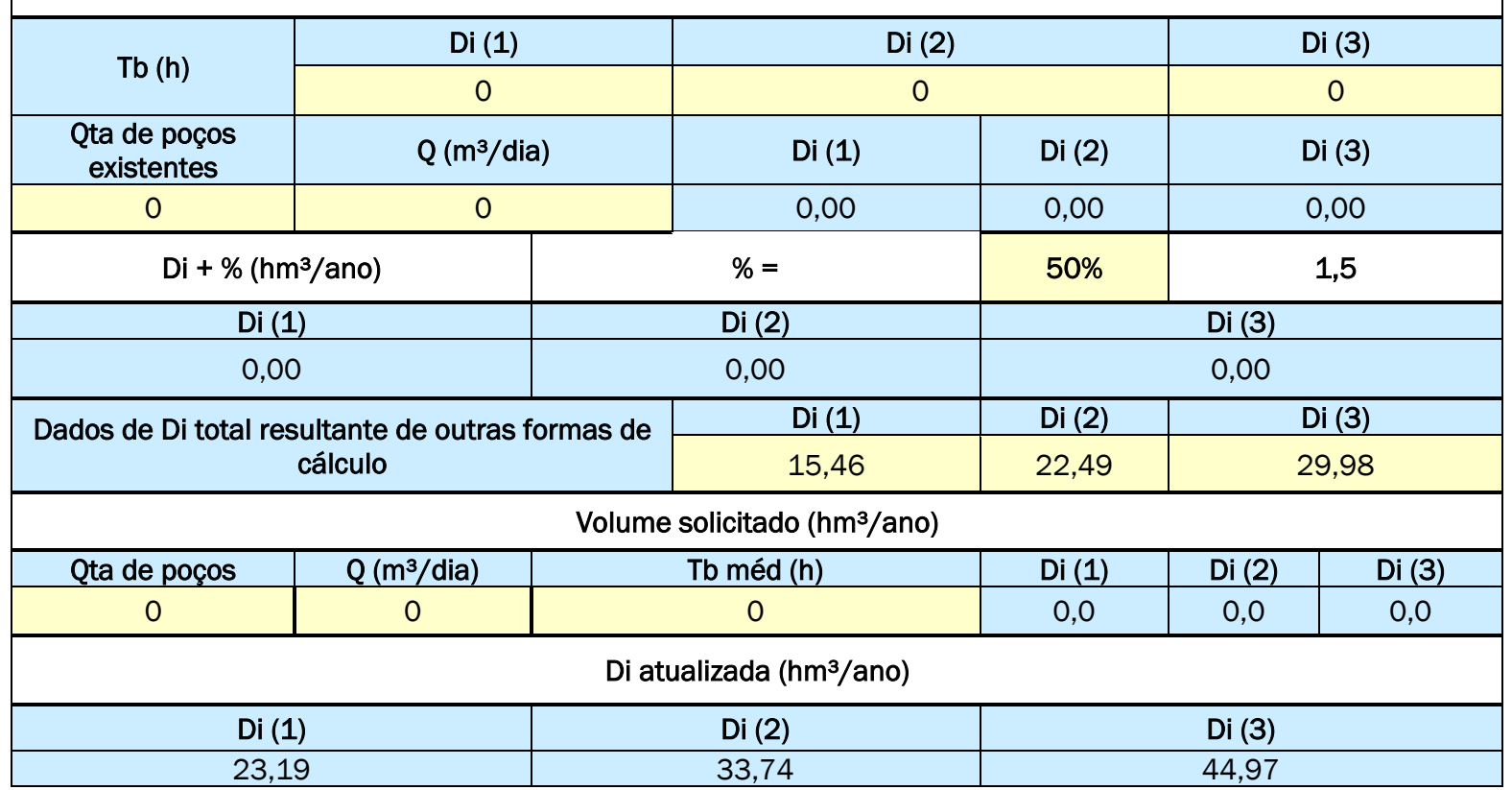

\subsection{Comprometimento do Potencial Hídrico Subterrâneo}

Os dois métodos analisados de balanço hídrico resultaram nos \% mais elevados de recarga, se comparados aos métodos de filtros digitais. Logo, os \% de comprometimento foram menores com a utilização dos métodos em questão. Já com a aplicação dos métodos dos filtros digitais, ocorreram duas situações:
- A primeira é referente a aplicação dos métodos de FP1 e $\mathrm{FI}$, os quais resultaram em \% de comprometimento muito próximos, devido à pouca diferença dos valores de recarga estimados entre esses dois métodos;

- A segunda é em relação ao método FP2, o qual resultou nos \% de comprometimento mais elevados e discrepante em relação aos demais métodos, devido ao baixo \% de recarga estimado. 
Os resultados oriundos das simulações realizadas pela CAL$\mathrm{POHID}$ indicam que as diferenças observadas entre os volumes de potencial gerados com os dados de recarga de cada método só se torna relevante quando considerado para a estimativa do potencial (\% sobre a recarga inferiores a 50\%). Tal situação pode ser observada no Quadro 8, onde é apresen- tado o módulo 2 da CALPOHID, no qual todos os graus de comprometimento foram baixos e com $\%$ inferiores a 35\% no cenário de $50 \%$ e a $17 \%$ no cenário de $100 \%$. Sendo assim, ambos os cenários de comprometimento não demandariam diferenças significativas nas medidas de gestão adotadas pelos gestores.

Quadro 8 - Estimativa dos \% e grau de comprometimento gerados no módulo 2

\begin{tabular}{|c|c|c|c|c|c|}
\hline \multicolumn{6}{|c|}{ Módulo 2} \\
\hline \multicolumn{6}{|c|}{ Comprometimento (\%) } \\
\hline \multicolumn{6}{|c|}{$15 \%$} \\
\hline Cenários & R1 & R2 & R3 & R4 & R5 \\
\hline Di (1) & $24,25 \%$ & $16,41 \%$ & $31,45 \%$ & $57,62 \%$ & $30,25 \%$ \\
\hline $\mathrm{Di}(2)$ & $35,28 \%$ & $23,87 \%$ & $45,75 \%$ & $83,82 \%$ & $44,01 \%$ \\
\hline Di (3) & $47,03 \%$ & $31,82 \%$ & $60,99 \%$ & $111,73 \%$ & $58,67 \%$ \\
\hline \multicolumn{6}{|c|}{$20 \%$} \\
\hline $\mathrm{Di}(1)$ & $18,19 \%$ & $12,31 \%$ & $23,59 \%$ & $43,21 \%$ & $22,69 \%$ \\
\hline $\mathrm{Di}(2)$ & $26,46 \%$ & $17,90 \%$ & $34,31 \%$ & $62,86 \%$ & $33,01 \%$ \\
\hline $\mathrm{Di}(3)$ & $35,27 \%$ & $23,87 \%$ & $45,74 \%$ & $83,80 \%$ & $44,00 \%$ \\
\hline \multicolumn{6}{|c|}{$25 \%$} \\
\hline Di (1) & $14,55 \%$ & $9,85 \%$ & $18,87 \%$ & $34,57 \%$ & $18,15 \%$ \\
\hline Di (2) & $21,17 \%$ & $14,32 \%$ & $27,45 \%$ & $50,29 \%$ & $26,41 \%$ \\
\hline Di (3) & $28,22 \%$ & $19,09 \%$ & $36,59 \%$ & $67,04 \%$ & $35,20 \%$ \\
\hline \multicolumn{6}{|c|}{$50 \%$} \\
\hline Di (1) & $7,28 \%$ & $4,92 \%$ & $9,44 \%$ & $17,29 \%$ & $9,08 \%$ \\
\hline Di (2) & $10,58 \%$ & $7,16 \%$ & $13,73 \%$ & $25,15 \%$ & $13,20 \%$ \\
\hline Di (3) & $14,11 \%$ & $9,55 \%$ & $18,30 \%$ & $33,52 \%$ & $17,60 \%$ \\
\hline \multicolumn{6}{|c|}{$100 \%$} \\
\hline Di (1) & $3,64 \%$ & $2,46 \%$ & $4,72 \%$ & $8,64 \%$ & $4,54 \%$ \\
\hline Di (2) & $5,29 \%$ & $3,58 \%$ & $6,86 \%$ & $12,57 \%$ & $6,60 \%$ \\
\hline Di (3) & $7,05 \%$ & $4,77 \%$ & $9,15 \%$ & $16,76 \%$ & $8,80 \%$ \\
\hline
\end{tabular}

Na utilização de $25 \%$ da recarga foi originada a primeira discordância entre os graus de comprometimento resultantes da aplicação de cada método. Esta ocorreu quando utilizados os dados de recarga de FP2, o qual resultou em grau de comprometimento médio para os cenários de Di (Tb 18 e 24h). Logo, a adoção do dado de recarga estimado por FP2, para a gestão dos recursos hídricos subterrâneos, induziria o gestor a um nível de atenção maior, levando o mesmo a agir com maior cautela, se comparado com o quadro de comprometimento gerados pelos dados de recarga estimados pelos demais métodos. No cenário de $20 \%$ da recarga, assim como ocorreu no cenário de $25 \%$, o único método que resultou em alteração no grau de comprometimento foi o FP2. No entanto, com apenas $5 \%$ a menos no volume do potencial, foram observadas 2 situações no quadro de comprometimento originado com o dado de recarga FP2:

- A primeira é a alteração do grau de comprometimento no cenário de Di (Tb 24h), o qual se elevou para crítico, com $\%$ de comprometimento superior a $80 \%$;
- O segundo é a aproximação do \% de comprometimento no cenário de Di (Tb médio e 18h), do limite superior para o enquadramento em grau médio e crítico, respectivamente;

Desta forma, adotar os dados do cenário de comprometimento diante da Di (24h) levaria à adoção de medidas de gestão mais restritivas, em relação as demandadas nos demais cenários oriundos dos outros métodos analisados.

Além das situações mencionadas, quando aplicado os dados de recarga dos métodos de FP1 e FI, o grau de comprometimento para o cenário mais crítico de Di ficaram a cerca de 5 e $6 \%$, respectivamente, do limite superior para o enquadramento em grau crítico. Portanto, para essa última situação, mesmo com grau de comprometimento se mantendo baixo, adotar algum desses cenários para a gestão dos recursos hídricos subterrâneos induziriam o gestor a uma maior atenção. 0 cenário mais restritivo de potencial, quando considerado $15 \%$ da recarga, resultou em alterações de grau de compro- 
metimento ante utilização de 3 métodos, sendo eles os métodos FP2, FP1 e FI. Nesses dois últimos, a alteração foi apenas no cenário mais crítico de $\mathrm{Di}$, sendo o grau de comprometimento médio com \% em torno dos $60 \%$ em ambos casos. 0 grau de comprometimento médio também foi gerado para o cenário menos crítico de Di quando utilizado o dado de recarga de FP2. Ainda no quadro de comprometimento de FP2, para os cenários de $\mathrm{Di}$ (Tb 18 e 24h), o grau de comprometimento foi crítico. Contudo, o cenário mais crítico de Di foi o único que gerou \% de comprometimento superior ao potencial total estimado, superando o mesmo em cerca de $11 \%$.

Os dados de recarga dos métodos de $\mathrm{BH} 1$ e $\mathrm{BH} 4$ resultaram em quadros de comprometimento baixo em todos os cenários simulados (Quadro 8), não demandando variações das medidas de gestão, atingindo no máximo cerca de 35\% da recarga (com o método $\mathrm{BH} 1$ ).

A ferramenta em questão se demonstrou útil para possibilitar uma visão mais ampla aos gestores diante dos quadros de comprometimento resultantes de dados de recarga estimados por diferentes métodos. A possibilidade de visualizar diferen- tes quadros de comprometimento pode auxiliar os gestores em qual caminho seguir:

- Para um caminho mais conservador: o método FP2;

- Para um caminho menos restritivo: o método BH4;

- Para um caminho mais ponderado entre todos os métodos: $\mathrm{BH} 1$.

O objetivo deste trabalho não é indicar o melhor ou pior método, mas sim a necessidade de variações nas medidas de gestão à frente da adoção de cada um. Além disso, demonstrar que, dependendo do valor de recarga utilizado na avaliação do potencial de recursos hídricos subterrâneos, poderão ocorrer variações significativas nos \% de comprometimento. 0 método que demanda variações nas medidas adotadas com maior frequência, em relação aos demais, é o FP2. Sendo assim, na figura 3, é apresentado o gráfico dos volumes restantes somente do método FP2, que foi o único a indicar no cenário mais crítico de Di um comprometimento superior ao potencial existente, de $4,72 \mathrm{hm}^{3}$. Os gráficos de volume restante indicam para os gestores os volumes que ainda teriam disponível para serem concedidos em novas outorgas.

Figura 3 - Gráfico do volume restante do potencial gerado no módulo 5

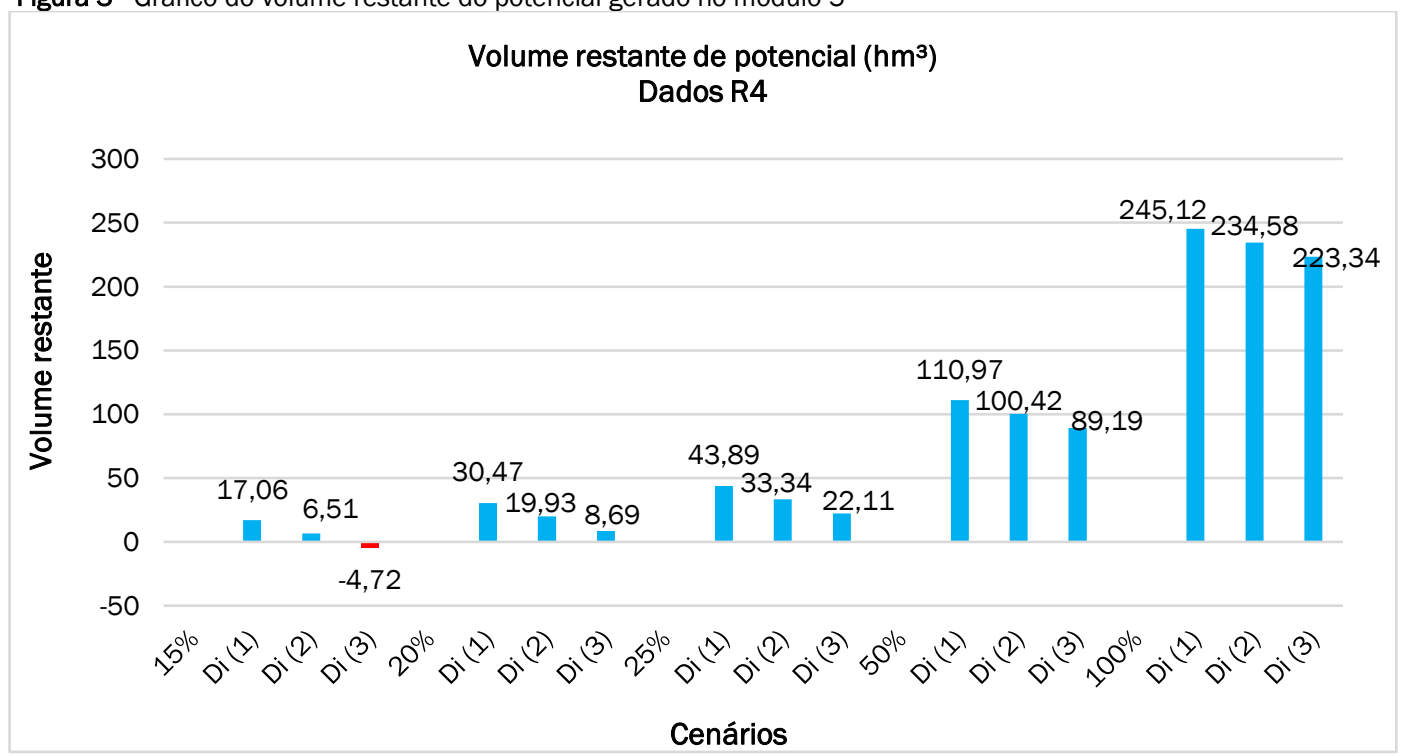

Cabe salientar, que os resultados da CALPOHID em si não podem ser utilizados como base única para a tomada de decisão. Assim sendo, os quadros de comprometimento devem ser verificados por meio de outros estudos, bem como pela instalação de redes de monitoramento de nível de água. No entanto, a CALPOHID se trata de uma ferramenta inovadora, que viabiliza de forma simples, objetiva e prática, os diferentes cenários que podem ocorrer em face das variações nos métodos de estimativa da recarga, se tornando de grande valia para a gestão dos recursos hídricos.

\subsection{Recarga versus Comprometimento do Potencial Hídrico Subterrâneo}

As diferenças existentes entre os \% de recarga (10 a 37\% da precipitação) refletiram em níveis diferentes nos \% de comprometimento estimados com cada método.

Na figura 4, é apresentado, em termos absolutos, os gráficos que relacionam as diferenças entre os \% de comprometimento no eixo y em relação a cada cenário analisado de tempo de bombeamento apresentado no eixo $\mathrm{x}$. Foram observadas as seguintes situações:

- A diferença máxima entre os \% de recarga ocorreu entre os métodos BH4 e FP2, a qual foi superior a $26 \%$. Tal diferença ocasionou em um \% de comprometimento de cerca de $12 \%$ superior em BH4 para o cenário mais crítico de Di, e de cerca de 9 e 6\% para os demais cenários de Tb 18h e médio, respectivamente; 
- A diferença mínima entre os \% de recarga ocorreu entre os métodos $\mathrm{FP} 1$ e $\mathrm{Fl}$, a qual foi inferior a $0,8 \%$, ocasionando \% de comprometimento distantes em no máximo 0,35\% para o cenário mais crítico de Di.

- O método que resultou em menores diferenças de \% de comprometimento em relação aos demais foi $\mathrm{BH} 1$, sendo essas de no máximo 2,24\% no cenário mais crítico de Di. A única exceção foi em relação ao método FP2, o qual apresentou \% de diferença maior. Isso ocorreu porque o método FP2 foi o que apresentou as maiores diferenças de recarga em relação aos demais métodos, a qual foi, em média, superior a $5 \%$.

Figura 4 - Relação das diferenças entre os \% de recarga estimada por cada método com as diferenças apresentadas entre os \% de comprometimento

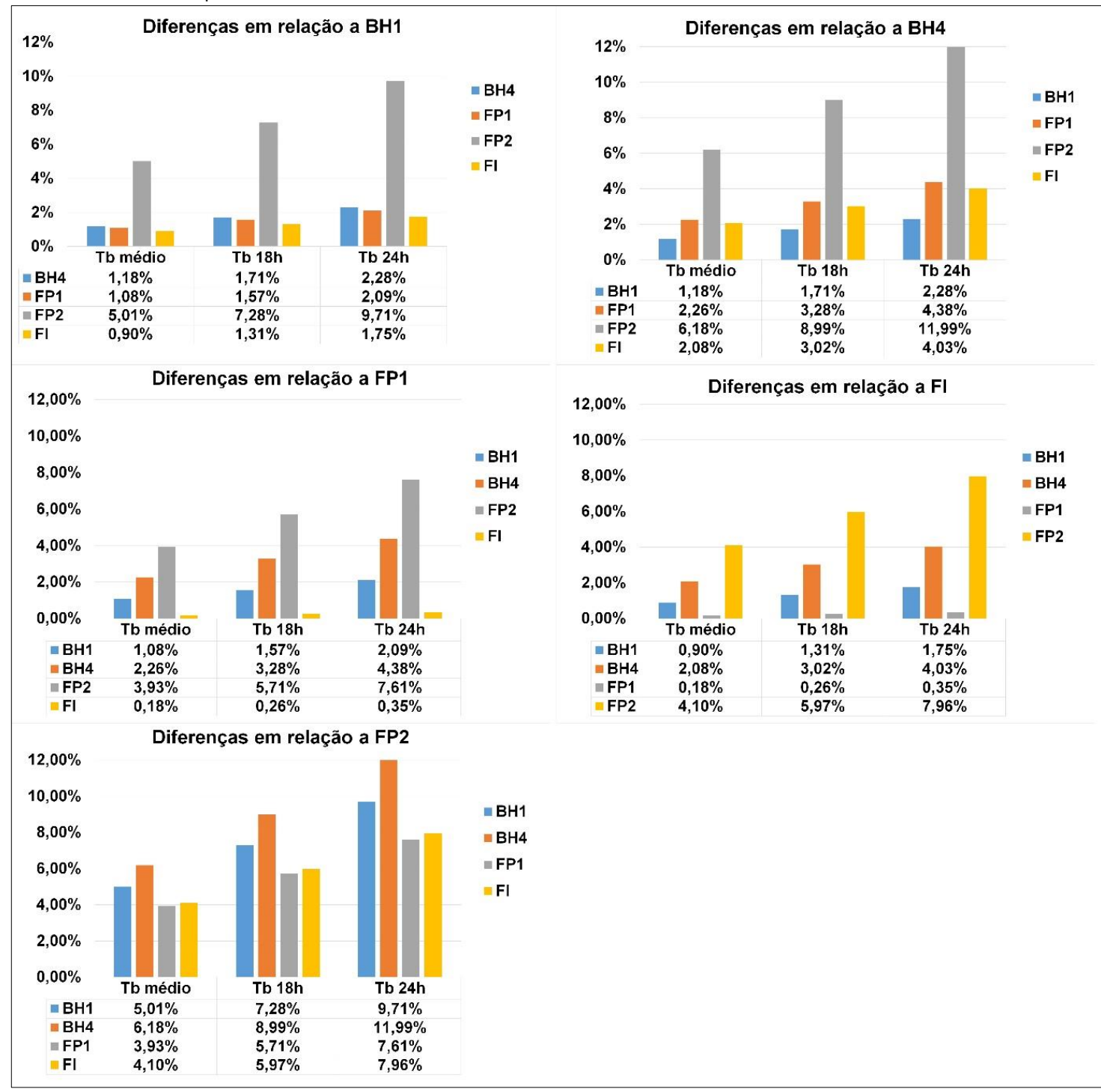

\section{CONCLUSÕES}

As diferenças dos potenciais entre os métodos foram menores entre os métodos FP1 e Fl. No entanto, em sua maioria, as diferenças foram significativas, levando em consideração que as mesmas poderiam atender no mínimo a $42 \%$ da Di em seu cenário mais crítico, já acrescido em 50\% na Di. Contudo, apenas nos cenários analisados inferiores a $50 \%$ da recarga foram identificadas alterações nos graus de comprometimento. Sendo que, nos cenários de 50 e 100\%, os graus de comprometimento foram todos baixos.

Dos 5 métodos simulados neste estudo, o FP2 seria o que mais demandaria variações nas medidas de gestão adotadas.
Além de método FP2, os métodos FP1 e FI também demandariam alterações nas medidas de gestão a serem adotadas para o cenário de $15 \%$ da recarga, considerando a Di (24h). As alterações mencionadas nas medidas de gestão ao adotar o método FP2, ou um dos dois cenários mencionados em FPI e Fl, seriam mais restritivas e/ou cautelosas se comparado as alterações necessárias diante do quadro de comprometimento dos demais métodos.

A ferramenta CALPOHID proposta se demonstrou eficiente para a comparação de cenários de potencial e comprometimento à frente de diferentes dados de recarga e disponibilidade hídrica instalada, porém, a precisão dos resultados ge- 
rados pela CALPOHID depende da qualidade dos dados inseridos no módulo 1. A tomada de decisão dos gestores não pode ser baseada unicamente pela análise de comprometimento realizada pela CALPOHID, sendo que os resultados da mesma devem ser verificados através da implantação de uma rede de monitoramento de níveis de água. Contudo, a ferramenta viabiliza uma visão mais ampla dos diferentes quadros de comprometimento gerados por diferentes métodos. Sendo assim, os gestores podem definir qual método adotar, se o mais conservador, menos restritivo ou ponderado. Tal possibilidade se torna de suma importância em sistemas aquíferos fraturados, os quais possuem maiores incertezas nos volumes de potenciais hídricos subterrâneos existentes.

Por fim, vale salientar, que a CALPOHID é uma ferramenta versátil, desenvolvida para ser aplicada em qualquer área de estudo, para ser inserida em diferentes sistemas aquíferos e considerando diferentes cenários de potencial e Di.

\section{REFERÊNCIAS}

ANA. Disponibilidades e demandas por recursos hídricos no Brasil, 2005.

ARNOLD, J. G. et al. Automated Base Flow Separation and Recession Analysis Techniques. Ground Water, [s. I.], v. 33, n. 6 , p. 1010-1018, 1995 . https://doi.org/10.1111/j.17456584.1995.tb00046.x

BORTOLIN, T.A. Estudo da recarga do Sistema Aquífero Serra Geral na bacia hidrográfica Taquari-Anta. Tese (Doutorado)Programa de Pós Graduação em Recursos Hídricos e Saneamento Ambiental, Instituto de Pesquisas Hidráulicas, Universidade Federal do Rio Grande do Sul, Porto Alegre, 2018. 220 p. https://doi.org/10.14295/ras.v0i0.28759

BORTOLIN, T.A; REGINATO, P.A.P; SCHNEIDER, V.E. Estimativa de recarga pelo método do balanço hídrico em uma bacia hidrográfica com ocorrência de aquíferos fraturados. In: XIX CONGRESSO BRASILEIRO DE ÁGUAS SUBTERRÂNEAS, 19. 2016. [Anais...]. Campinas-São Paulo, 2016.

COLLISCHONN, W.; FAN, F. Defining Parameters for Eckhardt's Digital Base Flow Filter. Hydrological Processes, v. 27, n, 18 p. 2614-2622, 2013. https://doi.org/10.1002/hyp.9391

CONSELHO ESTADUAL DE RECURSOS HÍDRICOS (São Paulo). Deliberação $n^{\circ}$ 052/CRH, de 15 de abril de 2005. Institui no âmbito do Sistema Integrado de Gerenciamento de Recursos Hídricos - SIGRH diretrizes e procedimentos para a definição de áreas de restrição e controle da captação e uso das águas subterrâneas.

COSTA, W. D. Avaliação das reservas, potencialidades e disponibilidade de aquífero. In: CONGRESSO BRASILEIRO DE ÁGUAS SUBTERRÂNEAS, 10., 1998. [Anais...]. São Paulo: ABAS. 1998. p. Trabalho 50, p 11.

DAEE. Elaboração do plano diretor de aproveitamento de recursos hídricos para a macrometrópole paulista. 2009. Disponivel em: http://www.daee.sp.gov.br/macrometropole/RA04_vol2_Nota_Tecnicas_05_08_09\%2809-0309\%29.pdf. Acesso em: 16 abril 2017.
DIAS, F.A. Caracterização Estrutural e Hidrogeológica do Sistema Aquífero Serra Geral (SASG) na região de Carlos Barbosa (RS). Trabalho de Conclusão de Curso. (Graduação) - Instituto de Geociências, Universidade Federal do Rio Grande do Sul. Porto Alegre, 2013. 58 p.

DUTRA, T. O.; REGINATO, P. A. R.; SILVA, T. C. Estimativa e comparação do potencial hídrico subterrâneo na Bacia Hidrográfica Taquari-Antas (RS). Revista Águas Subterrâneas, v. 33, n. 2, p. 146-158, 2019. https://doi.org/10.14295/ras.v33i2.29476

ECKHARDT, K. How to construct recursive digital filters for baseflow separation. Hydrological Process, v. 19, n. 2, p. $507-$ 515, 2005. https://doi.org/10.1002/hyp.5675

FENN, D. HANLEY, K., DE GEARE, T. Use of the water balance method for predicting leachate generation from solid waste disposal sites. US Environmental Protection Agency Report. 1975.

FREITAS, M.A.; BINOTTO, R.B.; NANNI, A.S.; RODRIGUES, A.L.M.; BORTOLI. Avaliação do Potencial Hidrogeológico, Vulnerabilidade Intrínseca e Hidroquímica do Sistema Aquífero Serra Geral no Noroeste do Estado do Rio Grande do Sul. Revista Brasileira de Recursos Hídricos, v. 17, n. 2, p. 31-41, 2012. https://doi.org/10.21168/rbrh.v17n2.p31-41

LYNE, V.D., HOLLICK, M. Stochastic time-variable rainfall runoff modeling. Hydro. and Water Resources Symposium Institution of Engineers, Perth, Australia, pp. 89-92, 1979.

MACHADO, J.L.F.; FREITAS, M.A. de. Projeto Mapa Hidrogeológico do Rio Grande do Sul: relatório final. Porto Alegre. CPRM. 65p. il. mapa. 2005.

Nathan, R.J., and McMahon. Evaluation of automated techniques for base flow and recession analyses: Water Resources Research, v. 26, no. 7, p. 1465-1473, 1990. https://doi.org/10.1029/WR026i007p01465

NRCS - Natural Resources Conservation Service. Chapter 10: Estimation of direct runoff from storm rainfall, Part 630, National Engineering Handbook. Washington, DC: US Department of Agriculture. Series C 222. Reidel, Dordrecht, p 223238, 2004.

REBOUÇAS, A. C.; Riccomini, C.; Ellert, N.; Duarte, U.; Melito, K.M.; Senf, L.A.; Souza, J.C.S., Diagnóstico Hidrogeológico da Região Metropolitana de São Paulo, Uso e Proteção. In: CONGRESSO BRASILEIRO DE ÁGUAS SUBTERRÂNEAS, 8., 1994. Recife. [Anais...] Recife, ABAS/DNPM/CPRM, p. 93-102.

REGINATO, P.A.R.; STRIEDER, A.J. Caracterização Hidrogeológica e Potencialidades dos Aquíferos Fraturados da Formação Serra Geral na Região Nordeste do Estado do Rio Grande do Sul. In: CONGRESSO BRASILEIRO DE ÁGUAS SUBTERRÂNEAS, 13., 2004. [Anais...]. Cuiabá, 2004. https://doi.org/10.25249/0375-7536.20063611322

REGINATO, P.A.R.; STRIEDER, A.J. Caracterização Estrutural dos Aquíferos Fraturados da Formação Serra Geral na Região Nordeste do Estado do Rio Grande do Sul. Revista Brasileira de Geociências, v. 36, n. 1, p. 13-22, 2006.

WAHNFRIED, I.; HIRATA, R. Comparação de Métodos de Estimativa de Recarga de Aquíferos em Uma Planície Aluvionar na Bacia Hidrográfica do Alto Tietê (São Paulo). Revista Brasileira 
de Recursos Hídricos, v. 10, n.2, p. 15-25, abr./jun. 2005. https://doi.org/10.21168/rbrh.v10n2.p15-25

STE - Serviços Técnicos de Engenharia. Plano da Bacia Hidrográfica Taquari Antas. 2011. Disponível em: https://www.sema.rs.gov.br/g040-bacia-hidrografica-do-riotaquari-antas. Acesso em: 01 mar. 2016.
STRECK, E. V.; KAMPF, N.; DALMOLIN, R. S. D. et al. Solos do Rio Grande do Sul. 2. ed. Porto Alegre: EMATER/RS, 2008. $222 \mathrm{p}$.

Todd. Groundwater hydrology. New York: John Wiley \& Sons, 1959. 319p. 